\title{
EVALUATION OF DIFFERENT CURING ENERGIES ON SOME PROPERTIES OF TWO RECENT RESINS COMPOSITE.
}

\author{
Ahmed M. Naguib*, Mohamed T. Fayed ${ }^{* *}$ and Saad El-Dein S. El-Ghzawy***
}

\begin{abstract}
Objective: Evaluation the curing capability of laser energy on new types of dental composites, in comparison to what's already available, is one of the main aims for this study, regarding the values of microhardness and the degree of conversion.

Methods: Fifty-six specimen prepared from two types of dental resin composites (SDR BulkFil@ and Ceram.X One $\subset$ resin composite), twenty-eight from each type of composite divided for four subgroups seven specimen for each. Samples were prepared in a Teflon split mold with fixed dimensions packed in incremental technique following the manufactures instructions, to produces each specimen in cylindrical shape with $4 \mathrm{~mm}$ diameters and $6 \mathrm{~mm}$ thickness. Three of each subgroup cured with different curing time using (SIROLaser@C Blue laser, Sirona) laser device for (5sec,10sec and 20sec) respectively on $445 \mathrm{~nm}$ wavelength and 500 mw power, and the last group cured by (BlueLEX LD-105, Monitex(C) LED curing unit for 20sec. The microhardness of top and bottom surfaces tested by Vicker's hardness testing machine (HV-1000DT) and then the specimens powdered and tested for the degree of conversion using Fourier transform infrared spectroscopy (Agilent Cary 630 FTIR).
\end{abstract}

Results: For SDR BulkFil composite resin group, top surface; It was found that 5-seconds laser cured subgroup recorded the highest mean \pm SD value of Vickers $\mu$-hardness by 20 -seconds LED cured subgroup. Regardless to composite resin type, it was found that, laser curing energy recorded higher (DC) percentage mean values than LED curing one.

Conclusions: SIROLaser Blue laser device ( $500 \mathrm{mw} / \mathrm{cm} 2$ and wavelength $445 \mathrm{~nm}$ ) have a significant capability of curing of Dental composites. Inanition, regardless to curing energies, it was concluded that Ceram.X One resin composite recorded statistically significant higher (DC) percentage mean values than SDR BulkFil composite resin.

KEYWORDS: Laser curing; SDR BulkFil; Ceram.X One; Degree of conversion; Microhardness

\section{INTRODUCTION}

Resin Composite dental restorations represent a unique class of biomaterials. These materials are presently limited by shrinkage and polymerization induced shrinkage stress, limited toughness, the presence of unreacted monomer that remains following the polymerization, and several other factors. Fortunately, these materials have been the focus of a great deal of research in recent years with the goal of improving restoration performance by changing the initiation system, monomers, fillers and their coupling agents by developing novel polymerization strategies ${ }^{(\mathbf{1})}$.

Effectiveness of the polymerization is one important meaning to obtain adequate physical properties. One limitation of photo-activated dental composite resins is that a hard-top surface is not an indication of adequate polymerization throughout the depth of restoration. Poorly polymerized composite resin can lead to undesirable effects, such as: gap formation, marginal microleakage, recurrent caries, adverse pulpal effects and ultimate failure of restorative procedure ${ }^{(2)}$.

\footnotetext{
* Demonstrator, Department of Biodental Materials, Faculty of Dental Medicine Al-Azhar University, Cairo. (Boys) ** Professor, Department of Operative Dentistry, Faculty of Dental Medicine Al-Azhar University, Cairo. (Boys) *** Lecturer, Department of Biodental materials, Faculty of Dental Medicine Al-Azhar University, Cairo. (Boys).
} 
Several attempts have been made to improve the mechanical properties by altering the composition of the material and the methods of composite polymerization. In the last decade, improvements have been made on the properties of dental composites including nanotechnology by reducing the filler particle size, increasing the filler volume to enhance wear resistance and polishing, and introducing the fillers that release fluoride. In addition, the development of the new Silorane monomers and modified urethane monomers were introduced in order to reduce polymerization shrinkage ${ }^{(3)}$.

Recently, a new category of resin composite, socalled bulk fill composite was introduced as bulk fill material and as filling in class I and II restorations. The particularity of the new material category is stated to be the option to place it in $4 \mathrm{~mm}$ thick bulks instead of the current incremental placement technique, without negatively affecting the degree of conversion, mechanical properties or cavity adaptation. However, in the clinical applications of the light cured composite resin, the efficiency of a light curing unit is crucial for obtaining the optimal degree polymerization and a successful outcome. With the research in the field of restorative materials, a need for an appropriate curing unit has always been felt. ${ }^{(2)}$

This is made possible by either a reduction in the filler content (Bulk-fill flowable composites), altering the filler matrix composition to improve the translucency of the material or by changing the photoinitiator system ${ }^{(4)}$. In addition, Bulkfill composites consist of ceramic fiber resin incorporated into the elongated filler network of about $100 \mathrm{~nm}$ in length. These materials have an increased depth of cure of up to $4 \mathrm{~mm}{ }^{(5)}$.

There are several factors affecting the curing outcome process, including the type of resin composite, shade and translucency, increment thickness, distance from the tip of the light cure unit, post- irradiation, the wavelength of the curing light used for polymerization, intensity of the curing light and irradiation type ${ }^{(6)}$.

It was reported that, the laser as a curing energy decreased curing time and further stated that all physical properties were enhanced when compared with conventional light curing units. The variables that control the depth and extent of cure include time of exposure, composite material, wavelength and intensity of the light, and particle size of the filler. Because laser light is intense, monochromatic, coherent, and collimated, it was thought that it might be a superior light source for photopolymerization of dental composite materials. ${ }^{(7)}$

Evaluation of the curing capability of laser energy on new types of dental composites, in comparison to what's already available, is one of the main aims for this study, regarding the values of microhardness and the degree of conversion as main parameters in evaluation of the end results gained by laser curing energy in comparison to LED curing one.

\section{MATERIALS AND METHODS}

The materials used in this study are two different types of new dental resin composites. The types of resin composite selected are Bulk fill resin composite (BulkFil SDR ${ }^{\odot)}$, and Nanohybrid Universal resin composite (Ceram.X One ${ }^{\mathcal{O}}$.

\section{Specimens grouping}

Twenty-eight specimens prepared from each type of composite subdivided into four groups seven in each one. Each subgroup subjected to different curing technique, the first one subjected to 20 seconds of laser curing, the second group subjected to 10 seconds of laser curing, the third group subjected to 5 seconds of laser curing, and the last group subjected to LED curing for 20 seconds. All specimens tested for microhardness on both top and bottom surfaces, and after the microhardness testing each group of specimens powdered to be tested for the degree of conversion. 


\section{Mold preparation:}

The mold was made of circular Teflon disk milled by specific diminutions $(30 \mathrm{~mm}$ in diameter and $6 \mathrm{~mm}$ in thickness), the mold was drilled to for three cylindrical holes vertically, aligned with the longest diameter of the disk and finally the disk split horizontally through the diameters of the aligned holes to make the split mold symmetrical. The two halves of the mold are assembled together with circumferential cupper ring with $35 \mathrm{~mm}$ diameter and $3 \mathrm{~mm}$ thickness.

\section{Samples preparation}

A Mylar strip was placed on a $5 \mathrm{~mm}$ thick glass slap and the Teflon mold was placed over it. The resin composite material was packed inside the mold. A second Mylar strip was placed over the resin composite and another glass slide $5 \mathrm{~mm}$ thickness was slightly compressed to extrude excess material and to keep the distance between the curing tip and the mold is fixed at $5 \mathrm{~mm}$.

For SDR BulkFil resin composite the material loaded into the mold through the nozzle tip in circular motion from the bottom of the mold up to the top, to allow proper adaptation against the mold walls and allow the air skip. The loading made in two steps (to $2 \mathrm{~mm}$ height mark from the bottom and curing, then to the top edge of the mold surface) to keep the thickness of each step not exceed $4 \mathrm{~mm}$ thickness.

For Ceram.X on resin composite the loading of composite into the mold made in successive oblique $2 \mathrm{~mm}$ thickness increments from the bottom of the mold to the top with curing each increment. The resin composite loaded and adapted against the mold walls using non-stick composite placement instrument.

\section{Curing of specimens:}

The types of curing systems selected are Laser system (SIROLaser Blue laser, Sirona ${ }^{\odot}$ ), and LED system (BlueLEX LD-105, Monitex ${ }^{\odot}$ ).

For conventional LED light curing, each specimen was irradiated according to the manufacturer instructions for $20 \mathrm{Sec}$. at $2000 \mathrm{mw} / \mathrm{cm}^{2}$ light intensity with LED curing unit (BlueLEX LD-105, Monitex ${ }^{\odot}$ )

For Laser Photoactivation the specimens were irradiated by SIROLaser Blue laser device (wavelength $445 \mathrm{~nm}$ and $500 \mathrm{mw} / \mathrm{cm} 2$ ). The Position of the light guide tip in contact with the glass slap on the top surface of the glass slap over the mold and held in place through the curing time intervals. Three different time intervals $(5 \mathrm{sec} ., 10 \mathrm{sec}$. and 20 sec.), seven specimens from each composite type for each time intervals.

\section{Vickers hardness test}

The Vickers hardness number (VHN) was determined on the top and the bottom surfaces for each specimen using a microhardness-testing machine* equipped with a diamond pyramidal

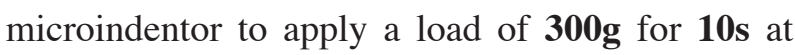
room temperature.

\section{Degree of conversion test}

All of the polymerized specimens $(n=7)$ of each group of cured composites was grinded into a fine powder through packing specimens into an empty and clean amalgam capsule contain small rough steel ball and using amalgamator vibrations for 2 minutes.

Fourier transform infrared spectroscopy** was used to evaluate the degree of conversion. This FTIR system uses the single reflection diamond attenuated total reflectance (ATR) specimen interface, which is requires no specimen preparation.

* (HV-1000DT) by Shanghai Daheng Optics and Fine Mechanics Co., Ltd.

** (Agilent Cary 630 FTIR) by Agilent technologies company www.agilent.com 
The calibration of the system performed first by placing the non-cured resin composite on the lens and diamond sensor adjusted to come in touch with the resin composite, and the system started to record the spectrum of the non-cured resin composite,

Then the spectrum for the cured resin composite obtained by placing small amount of the specimen's powder on the diamond sensor and using the specimen press to ensure good contact. The scanning cycle takes about 40 seconds including the correction scans to produce the full spectrum graph and all peaks values on the PC screen. The main functional peaks for chemical groups calculated automatically for further analysis.

\section{RESULTS}

Data analysis was performed in several steps. Initially, descriptive statistics for each group results. Two-way ANOVA was done for comparing resin composite and curing energy effect on different variable studied. One-way ANOVA followed by pair-wise Tukey's post-hoc tests were performed to detect significance between each composite subgroups and t-test for subgroups. Statistical analysis was performed using Asistat 7.6 statistics software for Windows (Campina Grande, Paraiba state, Brazil). $\mathrm{P}$ values $\leq 0.05$ are considered statistically significant in all tests.

\section{Vickers $\mu$-hardness}

The mean values and standard deviation of Vickers $\mu$-hardness (HV) test results for top, bottom surfaces and bottom to top $(\mathrm{B} / \mathrm{T})$ ratios of both dental resin composite photo-activated with different light curing energies are summarized in table (1) and graphically drawn in figure (1).

\section{For SDR BulkFil composite resin group}

\section{Top surface;}

It was found that 5 -seconds laser cured subgroup recorded the highest mean \pm SD value of Vickers $\mu$-hardness $(37.09 \pm 2.13 \mathrm{HV})$, followed by 20-seconds LED cured subgroup recorded mean $\pm \mathrm{SD}$ value of $(36.92 \pm 2.91 \mathrm{HV})$ then $\mathbf{1 0}$-seconds laser cured subgroup mean \pm SD value of

TABLE (1) Vickers $\mu$-hardness results (Mean values \pm SDs) for top, bottom surfaces and B/T ratios of both dental resin composite photo-activated with different light curing energy.

\begin{tabular}{|c|c|c|c|c|c|c|}
\hline \multirow{2}{*}{\multicolumn{2}{|c|}{ Variables }} & \multicolumn{2}{|c|}{ SDR BulkFil } & \multicolumn{2}{|c|}{ Ceram.X One } & \multirow{3}{*}{$\begin{array}{c}\text { Statistic } \\
\text { t-test } \\
\text { P value }\end{array}$} \\
\hline & & Top & Bottom & Top & Bottom & \\
\hline \multirow{6}{*}{ Laser } & 5 sec. & $37.09^{\mathrm{A}} \pm 2.13$ & $33.7^{\mathrm{A}} \pm 2.67$ & $84.37^{\mathrm{A}} \pm 7.9$ & $37.8^{\mathrm{B}} \pm 4.14$ & \\
\hline & $\mathrm{B} / \mathrm{T}$ ratio & \multicolumn{2}{|c|}{$91.06 \pm 7.51$} & \multicolumn{2}{|c|}{$44.93 \pm 4.19$} & $<0.0001^{*}$ \\
\hline & 10 sec. & $36.51^{\mathrm{A}} \pm 2.59$ & $34.19^{\mathrm{A}} \pm 2.72$ & $76.7^{\mathrm{A}} \pm 8.82$ & $38.24^{\mathrm{B}} \pm 4.36$ & Pvalue \\
\hline & $\mathrm{B} / \mathrm{T}$ ratio & \multicolumn{2}{|c|}{$93.71 \pm 4.61$} & \multicolumn{2}{|c|}{$50.75 \pm 9.53$} & $<0.0001^{*}$ \\
\hline & 20 sec. & $36.48^{\mathrm{A}} \pm 2.09$ & $33.59^{\mathrm{A}} \pm 2.89$ & $80.09^{\mathrm{A}} \pm 7.1$ & $39.52^{\mathrm{B}} \pm 5.4$ & Pvalue \\
\hline & $\mathrm{B} / \mathrm{T}$ ratio & \multicolumn{2}{|c|}{$92.1 \pm 6.03$} & \multicolumn{2}{|c|}{$49.36 \pm 5.02$} & $<0.0001^{*}$ \\
\hline \multirow{2}{*}{ LED } & 20 sec. & $36.92^{\mathrm{A}} \pm 2.91$ & $33.94^{\mathrm{A}} \pm 2.8$ & $78.19^{\mathrm{A}} \pm 9.08$ & $71.42^{\mathrm{A}} \pm 4.45$ & P value \\
\hline & $\mathrm{B} / \mathrm{T}$ ratio & \multicolumn{2}{|c|}{$92.19 \pm 6.73$} & \multicolumn{2}{|c|}{$91.35 \pm 10.07$} & $0.9739 \mathrm{~ns}$ \\
\hline ANOVA & P value & $0.9465 \mathrm{~ns}$ & $0.9733 \mathrm{~ns}$ & $0.2933 \mathrm{~ns}$ & $<0.0001 *$ & \\
\hline
\end{tabular}

Different letter in the same column indicating statistically significant difference $(p<0.05)$

*; significant $(p<0.05)$ $n s ;$ non-significant $(p>0.05)$ 


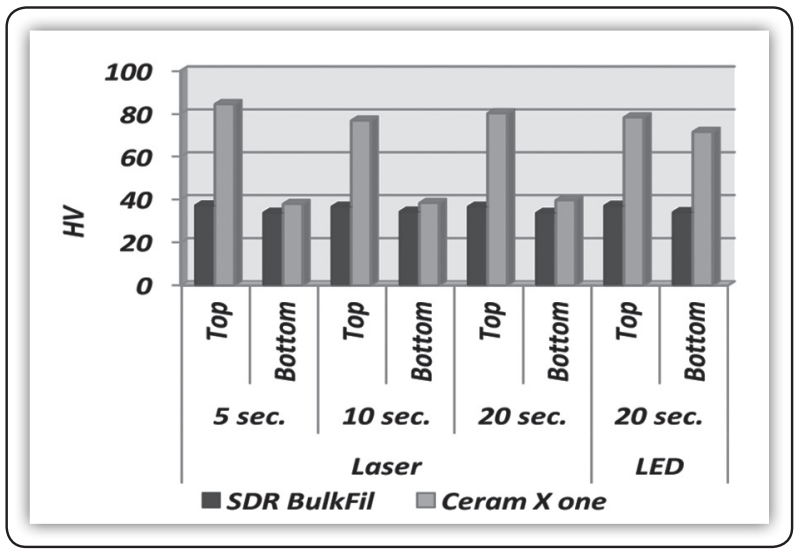

FIG (1) Column chart of the mean values of Vickers $\mu$-hardness for top and bottom surfaces of both dental resin composite photo-activated with different light curing energies

$(36.51 \pm 2.59 \mathrm{HV})$ meanwhile the lowest mean \pm SD value was recorded with $\mathbf{2 0}$-seconds laser cured subgroup was $(36.48 \pm 2.09 \mathrm{HV})$. The difference between different light curing energy subgroups was statistically non-significant as indicated by one-way ANOVA ( $\mathrm{F}=0.1219, \mathrm{P}=0.9465>0.05)$ as shown in table (1) and in figure (1).

\section{Bottom surface;}

It was found that 10-seconds laser cured subgroup recorded the highest mean $\pm \mathrm{SD}$ value of Vickers $\mu$-hardness $(34.19 \pm 2.72 \mathrm{HV})$, followed by 20-seconds LED cured subgroup recorded mean $\pm \mathrm{SD}$ value of $(33.94 \pm 2.8 \mathrm{HV})$, then 5 -seconds laser cured subgroup mean \pm SD value of $(33.7 \pm 2.67 \mathrm{HV})$. Meanwhile the lowest mean \pm SD value was recorded with $\mathbf{2 0}$-seconds laser cured subgroup was $(33.59 \pm 2.89 \mathrm{HV})$. The difference between different light curing energy subgroups was statistically non-significant as indicated by one-way ANOVA ( $\mathrm{F}=0.0744, \mathrm{P}=0.9733>0.05)$ as shown in table (1) and in figure (4).

\section{Degree of conversion (DC)}

The values of the degree of the conversion calculated by the verification of the absorption peak of $\mathrm{C}=\mathrm{C}$ bonds through the scanning spectrum, Spectra of paired un-polymerized and polymerized composite specimens were recorded in a transmission mode at room temperature, corrected by subtracting the background and then converted into the absorbance mode. Every scan per specimen were measured at a resolution of $4 \mathrm{~cm}^{-1}$. DC (\%) was calculated from the equivalent aliphatic (1638 $\left.\mathrm{cm}^{-1}\right) /$ aromatic $\left(1608 \mathrm{~cm}^{-1}\right)$ ratios of cured (C) and uncured (U) specimens according to the following expression: $\mathrm{DC}=(1-\mathrm{C} / \mathrm{U}) \mathrm{X} 100(\%)$.

The mean values and standard deviation of degree of conversion (DC) test results for both dental resin composite photo-activated with different light curing energies are summarized in table (2) and graphically drawn in figure (2).

\section{SDR BulkFil}

It was found that 5-seconds laser cured subgroup recorded the highest mean \pm SD value $(98.73 \pm 0.06 \%)$ followed by $\mathbf{2 0}$-seconds laser cured subgroup which recorded mean \pm SD value of $(97.84 \pm 0.04 \%)$ then 20-seconds LED cured subgroup mean \pm SD value of $(95.62 \pm 0.04 \%)$ meanwhile the lowest mean $\pm \mathrm{SD}$ value was recorded with $\mathbf{1 0}$-seconds laser cured subgroup was $(94.71 \pm 002 \%)$. The difference between different light curing energy subgroups was statistically significant as indicated by oneway ANOVA $(\mathrm{F}=8249, \mathrm{P}=<0.0001<0.05)$ as shown in table (2) and in figure (2).

\section{Ceram.X One}

It was found that 20-seconds LED cured subgroup recorded the highest mean $\pm \mathrm{SD}$ value of DC $(99.66 \pm 0.03 \%)$ followed by 10-seconds laser cured subgroup which recorded mean $\pm \mathrm{SD}$ value of $(99.55 \pm 0.01 \%)$ then 5 -seconds laser cured subgroup mean \pm SD value of $(99.53 \pm 0.05 \%)$ meanwhile the lowest mean $\pm \mathrm{SD}$ value was recorded with 20-seconds laser cured subgroup was $(98.06 \pm 0.09 \%)$. The difference between laser light curing energy subgroups and LED cured subgroup was statistically significant as indicated by one-way ANOVA ( $\mathrm{F}=1322.5, \mathrm{P}=<0.0001<0.05)$ as shown in table (2) and in figure (2). 
TABLE (2) Comparison of degree of conversion results (Mean values \pm SDs) between different light curing energy with both dental composite resin

\begin{tabular}{|c|c|c|c|c|}
\hline \multicolumn{2}{|c|}{$\begin{array}{c}\text { Variables } \\
\text { Mean } \pm \text { SD }\end{array}$} & SDR BulkFil & $\begin{array}{c}\text { Ceram.X } \\
\text { One }\end{array}$ & Statistics \\
\cline { 3 - 5 } & Mean \pm SD & t-test & \\
\hline \multirow{3}{*}{ Laser } & $\mathbf{5}$ sec. & $\mathbf{9 8 . 7 3}^{\mathrm{A}} \mathbf{\pm 0 . 0 6}$ & $99.53^{\mathrm{C}} \pm 0.05$ & $<0.0001^{*}$ \\
\cline { 2 - 5 } & $\mathbf{1 0}$ sec. & $\mathbf{9 4 . 7 1}^{\mathrm{D}} \mathbf{\pm 0 0 2}$ & $99.55^{\mathrm{B}} \pm 0.01$ & $<0.0001^{*}$ \\
\cline { 2 - 5 } & $\mathbf{2 0}$ sec. & $\mathbf{9 7 . 8 4}^{\mathrm{B}} \mathbf{\pm 0 . 0 4}$ & $98.06^{\mathrm{D}} \pm 0.09$ & $0.002^{*}$ \\
\hline \multirow{2}{*}{ LED } & $\mathbf{2 0}$ sec. & $\mathbf{9 5 . 6 2}^{\mathrm{C}} \mathbf{\pm 0 . 0 4}$ & $99.66^{\mathrm{A}} \pm 0.03$ & $<0.001^{*}$ \\
\hline ANOVA & P value & $<0.0001^{*}$ & $<0.0001^{*}$ & \\
\hline
\end{tabular}

Different letter in the same column indicating statistically significant difference $(p<0.05)$

*; significant $(p<0.05)$

$n s ;$ non-significant $(p>0.05)$

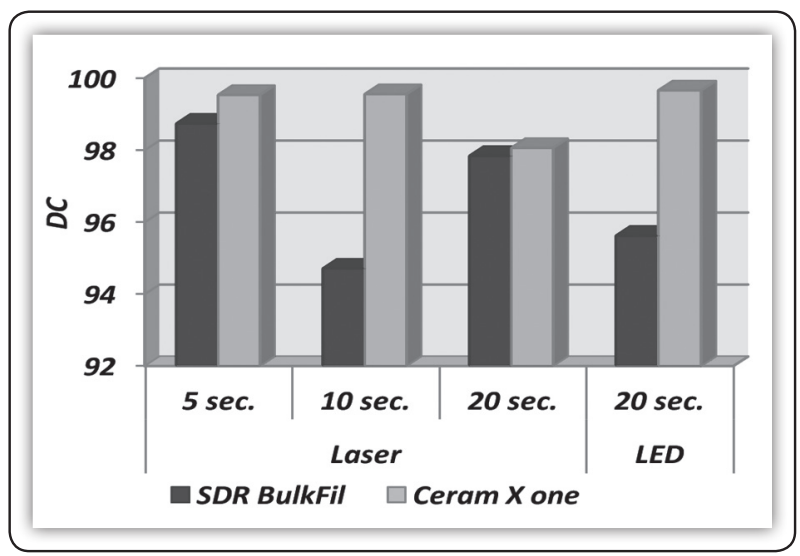

FIG (2) Column chart of the mean values of degree of conversion for different light curing energies with both dental resin composite

\section{DISCUSSION}

Evaluation the curing capability of laser energy on new types of dental composites in comparison to what is already available was one of the main aims for this study. In addition, selection of the curing units was one of the most challenging obstacles in this study, because of the limitation of the available Laser systems wavelength spectrum in relation to the spectrum we need to cure the composites, because the most of laser systems available are for the surgical uses only and the wavelength spectrum in a higher range than that used for resin composite curing.

Most RBCs contains camphorquinone (CQ) as the conventional photoinitiator system, which absorbs most efficiently at approximately $460-470 \mathrm{~nm}$; but some RBCs may contain alternative photoinitiators, such as diphenyl(2,4,6-trimethylbenzoyl) phosphine oxide (TPO), that absorb light most effectively within a lower wavelength range. These photoinitiators are often included as a substitute for CQ in commercial resin-based materials for esthetic restorations to reduce the yellow coloration ${ }^{(8)}$.

However, as the efficiency of these molecules depends on their absorption of light in a different wavelength range than CQ, newer Polywave LED curing lights that emit additional light at lower wavelengths near the UV range (i.e. $380-420 \mathrm{~nm}$ ) may be more optimal for light curing than the original monowave units that emit predominantly in the blue wavelength range (i.e. $420-495 \mathrm{~nm})^{(9)}$. This wavelength rage was optimum for the selected LED curing system for this study. But the lowest wavelength available on commercially available laser systems was Low-level laser-red nonsurgical wavelengths of 600 to $635 \mathrm{~nm}$ (for photobiomodulation) and $655 \mathrm{~nm}$ (for caries detection)- ${ }^{(\mathbf{1 0})}$.

Therefor in the current study, it was a demand to search for laser system provides a wavelength spectrum from $(420-495 \mathrm{~nm})$ to be in the curing spectrum of dental composite. Finally, we founded (SIROLaser Blue $^{\odot}-$ SIRONA, Germany) laser system as the first dental diode laser to have a blue, an infrared, and a red diode. That contain a Blue diode laser produces $445 \mathrm{~nm}$ wavelength and $0.2-3$ watts power. So, the one of the challenging points of this thesis is to use this system for curing of dental composites on $445 \mathrm{~nm}$ wavelength and compare the results with the already available LED curing units, 
depending on the microhardness and the degree of conversion.

The most commonly used photosensitizer in $\mathrm{RBCs}$ is camphorquinone (CQ). Alternative photoinitiators that are not as chromogenic as CQ are used by some RBC manufacturers and are found in the SDR Bulk Fill used in this study. These alternative photoinitiators, such as the monoacylphosphine oxide initiators (e.g., TPO) and derivatives of dibenzoyl germanium, have peak absorbance values below $420 \mathrm{~nm}^{(\mathbf{1 1})}$.

As these photoinitiators will not be efficiently activated by monowave LED-based LCUs that deliver light mostly in 445- $480 \mathrm{~nm}$ spectral range, a broader spectral emission LED unit $\left(\right.$ Monitex $^{\odot}$, BlueLEX LD-105) was used in this study with wavelength range $(420-490 \mathrm{~nm})$. The light output from this LCU remained stable over the 20s exposure time and had two peaks in the spectral emission, one at $455 \mathrm{~nm}$ and the other at $465 \mathrm{~nm}$.

\section{Microhardness}

Evaluation of micro-hardness is used widely as a test to assess the curing of the composites and the efficiency of light sources ${ }^{(\mathbf{1 2})}$. The surface microhardness of resin composites has been used to evaluate the efficiency of the light cure unit and to evaluate the extent of polymerization indirectly ${ }^{\left({ }^{(}\right)}$.

As shown in the results of Vickers $\mu$-hardness for SDR BulkFil resin composite group Top surface, it was found that 5-seconds laser cured subgroup recorded the highest mean value of Vickers $\mu$-hardness followed by 20 -seconds LED cured subgroup. Also, the results For Vickers $\mu$-hardness Ceram.X One resin composite group top surface, it was found that 5-seconds laser cured subgroup recorded the highest mean value of Vickers $\mu$-hardness followed 20-seconds LED cured subgroup mean value. This may regarded as an improvement point for surface hardness for both types of composites in this study,(SDR BulkFil composite and Ceram.X one composite) using laser curing technique, as we can reach the highest top surface microhardness in 5 seconds only with laser than 20seconds with the LED this may shorten the curing time by $75 \%$ when we use laser with types of composites in this study instead of LED and this findings agrees with ${ }^{(13)}$.

According to a study reported that, (14) This improvement of hardness values may be due to the hardness of this composite influenced by the crosslinking and network formation taking place during setting. This network formation occurs after an initial stage of polymer chain propagation.

Although these alternative photoinitiator in SDR BulkFil composite is more reactive than CQ, due to the effects of filler particle size and increased Rayleigh scattering ${ }^{\left({ }^{15}\right)}$, few of the lower wavelength photons will reach the bottom of 6-mm thickness of the $\mathrm{RBC}$ and the alternative photoinitiators will have to be activated by the less efficient longer wavelengths of light. This may explain why a previous observation found that the micro hardness of the bottom surface cure of filled CQ-based materials can be significantly greater than that of TPO-based materials using a LCU that delivered the greatest light output in the $450-500 \mathrm{~nm}$ range (16). But this postulation didn't agree with This study result, as it founded that, regardless to curing energies it was found that SDR BulkFil composite resin recorded statistically significant higher $(\mathrm{B} / \mathrm{T})$ ratio than Ceram $\mathrm{X}$ one composite resin. Therefore, I postulated that, these discrepancies might be attributed mainly to the composition of the materials, which influences the translucency, and as a result the energy density that reach the lower layers of the materials. It is important to mention that the microhardness of an RBC material does not reflect only the extent of polymerization, but other factors such as filler content and filler size, largely affect hardness results. Consequently, the comparisons among the tested materials concern their hardness as a mechanical property. ${ }^{(17)}$ 
According to a study ${ }^{(18)}$ showed that, the top surfaces of resin composite materials show higher micro-hardness values compared to the bottom surfaces. Besides that, there are some factors like the interactions of the matrix-filler which highly influence the micro-hardness and wear behavior of the materials. So, the micro-hardness of composites decreased with increasing depth of composite ${ }^{(19)}$. This point agree with the results shows the significant variance between the hardness values of the bottom surface for the two types of composites on this study. And also, it shows that, the laser curing energy couldn't improve the hardness of the composite at $4 \mathrm{~mm}$ thickness of Ceram.X in relation to the hardness values of bottom surface for SDR BulkFil composite.

In comparison of results Vickers hardness founded on laser against LED curing energy for each type of composite it was founded that: SDR BulkFil Composite resin, it was noted that LED cured SDR BulkFil composite resin recorded higher $\mathrm{B} / \mathrm{T}$ ratio than laser cured one. The difference in $\mathrm{B} / \mathrm{T}$ ratio between both energies was statistically nonsignificant. So, regarding to the type of composite laser curing light device cured photoactivated SDR BulkFil dental composite materials and provided a hardness value as efficient as conventional LED light curing devices but with shorter time.

For Ceram.X One resin Composite it was noted that, LED cured Ceram.X One resin composite recorded higher $\mathrm{B} / \mathrm{T}$ ratio than laser cured one. It could be explained by the higher density achieved in the densely compacted cross-network of dental resin Composite. However, this is not the only factor influencing the hardness of dental composite resins and fillers are recognized as more influential. The exceptions are microfilled composites with prepolymerized filler particles and higher amount of organic matrix ${ }^{(20)}$, similar to Ceram.X material to in this study. Although it contains nanofiller particles, which characterizes it as a nanohybrid resin composite, it also contains prepolymerized resin fillers, which are consisted of fillers embedded in resin, polymerized and milled to obtain a desired particle size. Therefore, prepolymerized fillers never achieve as high microhardness values as the composites without prepolymerized particles (21), which is in agreement with our results.

The results of Vickers hardness for both SDR BulkFil and Ceram.X resin composite group for Top surface; it was found that, 5-seconds laser cured subgroup recorded higher values than 10 -seconds laser cured subgroup. The explanation of this results may be due to the hardness influenced by the crosslinking and network formation taking place during initial setting stages ${ }^{(22)}$. But with the continuity of exposure to laser in the continuous mood, may leads to heat generation on the outermost layer of the composite, this heat transmission to the material may be responsible for this decrease of hardness, because heat increases mobility of the monomers by decreasing the cross liking and change the filler distribution on the outer top layer facing the laser source. This hypothesis agrees with some studies ${ }^{(23,19)}$.

\section{Degree of conversion}

As it was mentioned before, one of the aims of this study was to evaluate the curing capability of laser energy on new types of dental composites in comparison to what is already available, through the values of micro hardness and the degree of conversion.

For a dental restorative resin composite, it has appeared to be a good correlation between increasing hardness and increasing degree of conversion during the setting reaction. However, the acquisition of hardness chronologically lags behind the conversion of carbon double bonds (24) A much greater percentage of carbon double bonds are reacted to form polymer chains than are reacted to crosslink existing chains. Therefore, the greatest increase in hardness occurs during a period in which very subtle changes in DC take place, i.e. after $85 \%$ of the total conversion has been achieved ${ }^{(24)}$. 
Also it ${ }^{(25)}$ had reported that, The criteria of the bottom to the top hardness ratio from $0.80-0.90$ have been used as a predictor for adequate depth of curing at a specific specimen thickness, and this criteria means that the ratio of bottom to top surface micro-hardness is $80 \%$ or more will indicate adequate curing . Despite the correlation between hardness and DC during setting, a specific hardness value cannot be correlated to a specific DC when comparing different resin formulations.

According to ${ }^{(26)}$ the study that aimed firstly to investigate and compare the degree of conversion and microhardness of three modern dental composite materials. The second aim was to assess the validity of the earlier statement that degree of conversion can be accurately measured by means of microhardness instead of spectroscopic methods. Vickers microhardness and the DC were measured and the comparison of the data demonstrated that there was no correlation between them. This demonstrates the reason for measuring the degree of conversion separately on the types of resin composites under investigations on this study.

The predominant base monomer used in commercial dental composites is bisphenol A-glycidyl methacrylate (Bis-GMA), which is also present in all materials tested in this study. Because of its large molecular size and rigid structure, Bis-GMA has high viscosity, providing lower polymerization shrinkage, more rapid hardening and production of stronger and stiffer resins ${ }^{(27)}$. On the other side, its low mobility does not allow it to achieve high DC values. Therefore, Bis-GMA has to be mixed with diluent monomers of low viscosity, such as triethyleneglycol dimethacrylate (TEGDMA), urethane dimethacrylate (UDMA) or bisphenol A polyethylene glycol diether dimethacrylate (Bis-EMA) in order to achieve acceptable levels of polymerization ${ }^{(20)}$. Besides, the material has the highest filler volume ( as Ceram.X) of tested materials, which has probably contributed to increased light scattering, higher viscosity of the composite paste, therefore lower monomer mobility and consequently lower DC ${ }^{(28)}$. This explains the difference in the degree of conversion results between the two types of composite tested in this study regardless the curing energy differences.

However, for the tested composite materials, it is obvious that Ceram.X, exhibits a greater degree of conversion than SDR, regardless of the light source used. Ceram.X and SDR BulkFil can be seen as differing in the amount of inorganic and organic filler and in the size of filler particles, which points to the fact that the degree of conversion greatly depends not only on the intensity of the light source but also on the composition of the composite material. But, in the other side this results didn't agree with the studied said that, bulk-fills display a higher translucency than conventional resin composites ${ }^{(29)}$. As light transmission is strongly linked to material opacity ${ }^{(30)}$. Higher translucency can also be achieved by reduction in filler content as in SDR BulkFil ${ }^{(31)}$. For experimental resin composites it has been demonstrated that increasing the filler-to-matrix ratio progressively decreases conversion ${ }^{(32)}$.

The results of the degree of conversion for SDR BulkFil was shown that 5-seconds laser cured subgroup recorded the highest mean value followed 20 -seconds LED cured subgroup mean value and the difference between different light curing energy subgroups was statistically significant. This also show the efficiency of laser curing energy in comparison to LED and the reflection on the resulted degree of conversion.

Nevertheless, the results of the degree of conversion for SDR BulkFil was shown that, 5seconds laser cured subgroup recorded higher mean value than 20 seconds laser cured subgroup. Therefore, I postulate that with the continuity of irradiation with laser, the high energy of laser may lead to degradation of the formed monomer-tomonomer bond so the recorded final numbers of formed bonds will decrease as shown in the results. 
The results of the degree of conversion of the Ceram.X One resin composite shown that, the DC values for LED curing for 20 seconds was so close to that of the 10 seconds of Laser curing this reflect the ability of the laser energy to cure the Ceram.X One resin composite in the half of the time needed to have the same results by LED.

Nevertheless, the results of the degree of conversion for Ceram.X One was shown that, 10seconds laser cured subgroup recorded higher mean value than 20seconds laser cured subgroup. Therefore, I postulate that with the continuity of irradiation with laser, the high energy of laser may lead to degradation of the formed monomer-to-monomer bond so the recorded final numbers of formed bonds will decrease as shown in the results.

\section{CONCLUSIONS}

Within the limitation of the present study, it could be concluded that, SIROLaser Blue laser device (500 mw/cm2 and wavelength $445 \mathrm{~nm}$ ) capable of curing Dental composites. In addition, there is non-significant difference in hardness $\mathrm{B} / \mathrm{T}$ ratio between both LED curing and laser curing on SDR BulkFil composite. Regardless to curing energies, it was concluded that Ceram.X One resin composite recorded statistically significant higher (DC) percentage mean values than SDR BulkFil composite resin.

\section{REFERENCES}

1. Cramer N, Stansbury J, Bowman C. Recent advances and developments in composite dental restorative materials. Journal of Dental Research. 2011;90(4):402-16.

2. Czasch P, Ilie N. In vitro comparison of mechanical properties and degree of cure of bulk fill composites. Clinical Oral Investigations. 2013;17(1):227-35.

3. Finan L, Palin WM, Moskwa N, McGinley EL, Fleming GJ. The influence of irradiation potential on the degree of conversion and mechanical properties of two bulk-fill flowable RBC base materials. Dental Materials. 2013;29(8):906-12.
4. Ferracane JL, Pfeifer CS, Hilton TJ. Microstructural Features of Current Resin Composite Materials. Current Oral Health Reports. 2014;1(4):205-12.

5. Jackson R. New posterior composite materials improving placement efficiency. Compendium of continuing education in dentistry (Jamesburg, NJ: 1995). 2012;33(4):292.

6. Alrahlah A, Silikas N, Watts D. Post-cure depth of cure of bulk fill dental resin-composites. Dental Materials. 2014;30(2):149-54.

7. Rode KM, de Freitas PM, Lloret PR, Powell LG, Turbino ML. Micro-hardness evaluation of a micro-hybrid composite resin light cured with halogen light, light-emitting diode and argon ion laser. Lasers in medical science. 2009;24(1):87-92.

8. Alvim HH, Alecio AC, Vasconcellos WA, Furlan M, de Oliveira JE, Saad JR. Analysis of camphorquinone in composite resins as a function of shade. Dental Materials. 2007;23(10):1245-9.

9. Neumann MG, Schmitt CC, Ferreira GC, Corrêa IC. The initiating radical yields and the efficiency of polymerization for various dental photoinitiators excited by different light curing units. Dental Materials. 2006;22(6):576-84.

10. Coluzzi DJ, Convissar RA, ROSHKIND DM. Laser fundamentals. Principles and practice of laser dentistry. 2011:12-26.

11. Randolph LD, Palin WM, Bebelman S, Devaux J, Gallez B, Leloup G, et al. Ultra-fast light-curing resin composite with increased conversion and reduced monomer elution. Dental Materials. 2014;30(5):594-604.

12. Yaman BC, Efes BG, Dörter C, Gömeç Y, Erdilek D, Büyükgökçesu S. The effects of halogen and light-emitting diode light curing on the depth of cure and surface microhardness of composite resins. Journal of Conservative Dentistry. 2011;14(2):136.

13. Hofmann N, Hugo B, Klaiber B. Effect of irradiation type (LED or QTH) on photo-activated composite shrinkage strain kinetics, temperature rise, and hardness. European journal of oral sciences. 2002;110(6):471-9.

14. Dickens SH, Stansbury J, Choi K, Floyd C. Photopolymerization kinetics of methacrylate dental resins. Macromolecules. 2003;36(16):6043-53.

15. Masotti AS, Onófrio ÁB, Conceição EN, Spohr AM. UVvis spectrophotometric direct transmittance analysis of composite resins. Dental materials. 2007;23(6):724-30. 
16. Leprince J, Hadis M, Shortall A, Ferracane J, Devaux J, Leloup G, et al. Photoinitiator type and applicability of exposure reciprocity law in filled and unfilled photoactive resins. Dental Materials. 2011;27(2):157-64.

17. Dionysopoulos D, Tolidis K, Gerasimou P. The Effect of Composition, Temperature and Post-Irradiation Curing of Bulk Fill Resin Composites on Polymerization Efficiency. Materials Research. 2016(AHEAD):0-

18. Coffey O, Ray NJ, Lynch CD, Burke FM, Hannigan A. In vitro study of surface microhardness of a resin composite exposed to a quartz-halogen lamp. Quintessence international. 2004;35(10).

19. Manhart J, Kunzelmann K-H, Chen H, Hickel R. Mechanical properties and wear behavior of light-cured packable composite resins. Dental Materials. 2000;16(1):33-40.

20. Ferracane JL. Resin composite - state of the art. Dental materials. 2011;27(1):29-38.

21. Blackham J, Vandewalle K, Lien W. Properties of hybrid resin composite systems containing prepolymerized filler particles. Operative dentistry. 2009;34(6):697-702.

22. Horie K, Otagawa A, Muraoka M, Mita I. Calorimetric investigation of polymerization reactions. V. Crosslinked copolymerization of methyl methacrylate with ethylene dimethacrylate. Journal of Polymer Science: Polymer Chemistry Edition. 1975;13(2):445-54.

23. Harrington E, Wilson H. Determination of radiation energy emitted by light activation units. Journal of oral rehabilitation. 1995;22(5):377-85.

24. Issa Y, Watts DC, Boyd D, Price RB. Effect of curing light emission spectrum on the nanohardness and elastic modulus of two bulk-fill resin composites. Dental Materials. 2016;32(4):535-50

25. Rouhollahi M, Mohammadibasir M, Talim S. Comparative depth of cure among two light-cured core build-up composites by surface vickers hardness. Journal of dentistry (Tehran, Iran). 2012;9(3):255.

26. Marovic D, Panduric V, Tarle Z, Ristic M, Sariri K, Demoli $\mathrm{N}$, et al. Degree of conversion and microhardness of dental composite resin materials. Journal of molecular structure. 2013;1044:299-302.

27. Amirouche-Korichi A, Mouzali M, Watts DC. Effects of monomer ratios and highly radiopaque fillers on degree of conversion and shrinkage-strain of dental resin composites. Dental Materials. 2009;25(11):1411-8.

28. Hadis M, Leprince J, Shortall A, Devaux J, Leloup G, Palin W. High irradiance curing and anomalies of exposure reciprocity law in resin-based materials. Journal of dentistry. 2011;39(8):549-57.

29. Bucuta S, Ilie N. Light transmittance and micro-mechanical properties of bulk fill vs. conventional resin based composites. Clinical Oral Investigations. 2014;18(8):1991-2000.

30. Shortall A. How light source and product shade influence cure depth for a contemporary composite. Journal of oral rehabilitation. 2005;32(12):906-11.

31. Lee Y-K. Influence of filler on the difference between the transmitted and reflected colors of experimental resin composites. Dental Materials. 2008;24(9):1243-7.

32. Halvorson RH, Erickson RL, Davidson CL. The effect of filler and silane content on conversion of resin-based composite. Dental Materials. 2003;19(4):327-33. 